\title{
Optimized Ranking Based Recommender System for Various Application Based Fields
}

\author{
Sandhya Verma1 and Amit Kumar Manjhvar2 \\ Research Scholar1, Assistant Professor2 \\ sandhya.verma2508@gmail.com,amitkumar@mits.in
}

\begin{abstract}
To find the fascinating example, distinguish web client conduct, enhance the business procedure, anticipate web client desire, we are utilizing web utilization mining making utilization of affiliation standard mining To find the relation between the data item, we are using the association rule mining, which is an important field of data mining. Information is assembled on the web server as web log record in web usage mining. A different number of website visitor access the website that is why accessing of web logs and identifying relationships among these logs becomes a complex task because of rapid growth of web log files. To observe the relationship between the log records before applying the affiliation lead some preprocessing works are expected to diminish the uproarious information of web log documents. Multiple researchers done the different type of works on the WCM and WUM to enhance the competence of the website by providing innovative methods and and this paper gives a review about the current works done on WUM by the scientists.
\end{abstract}

Keywords: Web usage mining, web mining, data mining

\section{Introduction}

Data mining announces to obtain or "mining" knowledge from data in the vast amount. DM can be characterized as the mining of useful patterns from a type of data. DM is the extraction of non trivial, potentially useful information, implicit, previously unknown and pattern from the data from a large database.

Data mining is, basically, posing questions and finding the patterns related to them. The data can find out by using various mining techniques. In the modern era, WWW has impacted all aspects of our lives. The web has the same kind of attributes which make the mining of valuable information and knowledge a challenging task.

\section{Web Mining}

WM plays a very important role in finding the frequent pattern from World Wide Web is the source of the enormous amount of data, but every time it does not provide authentic data, so we use web mining that rarefy data. WM is to apply the DM technique to extract and uncover knowledge from the web documents services, i.e. web data. Web mining is the field of data mining. There are mainly three categories of web mining:

\subsection{Web Structure Mining}

This mining provides the relationship between the two web pages. In this, we establish the link in the middle web pages so that we can navigate one page to another. At the inter document level the WSM mining tries to explore the link structure of the hyperlink. For example - In any government site there are many jobs related hyperlink so that we can easily navigate to that site.

a) Hyperlinks 
A hyperlink is an architectural unit that hooks up to a location in a web page to a distinct location, either an alternate page or inside of the same website page. An intrarecord hyperlink which unites with an alternate part of the same page, and an interdocument hyperlink which connects two distinct pages.

b) Document Structure

Record Structure Mining endeavors have concentrated on naturally separating archive object model (DOM) structures out of reports. The website page substance can likewise be framed in a tree structure group, taking into account the different XML and HTML labels inside of the pages.

\subsection{Web Content Mining}

In this technique, filtering is done based on customer's interested item. From the ancient database, web pages are recommended very rapidly for the user. In that database different content of items are added that the user has used in the antique time and/or user's preferences and personal information. The data files of the user's can be assembled by using item rating, response to questions, or the user's navigation information to assume the user's interest and preferences. By using this filtering method, recommendation can be completed mainly from the past experience and available database of the website visitor.

WCM is also called a Snare content mining. The content mining specify to the originality of valuable information from web contents which consist of transcript, illustrations, videocassette, etc. It includes removal of structured data from snare pages, credentials, similarity and assimilation data's with comparable meaning, view removal from online sources, and concept hierarchy, responsiveness incorporation.

\subsection{Web Usage Mining}

The third piece of web mining is the web usage mining. In the web usage mining collection of information is allowed which is access to the web pages from the web. From the web server this information is gathered as the web logs. To access the web pages it provides the path. This web mining empowers the online business to give the best get to courses to benefits or other notice. The web utilization digging information takes into account the best get to ways to these entries, when an organization publicizes for administrations gave by different organizations. WUM is not only helpful for the e-business whose business is based singly on the traffic provided through a search engine, but also helpful for the web based business like online marketing. This WM also helps to bunch up the information which is important for the customer visiting the site. For the company's productivity flow this enables deeply log to complete the analysis.

WUM intends to mine the information from the web access conduct of the clients in the meantime, as they are skimming and investigating through the Web. The fundamental purpose of appreciation the course slant of the visitors is to enhance the way of electronic exchange organizations (e-business), to tweak the Web passageways, to customize the Web gateways or to enhance the Web server executes and web structure. 


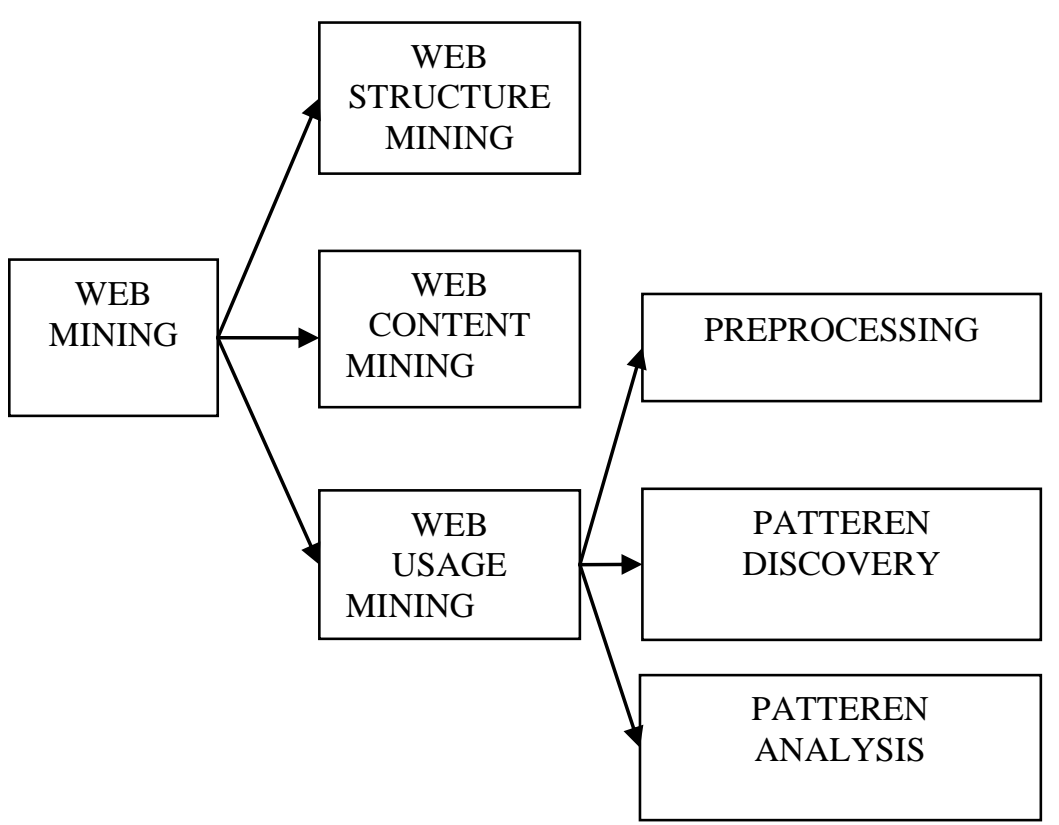

Figure 1. Techniques of Web Mining

There are three principle utilizes for mining as a part of this style.

1. Usage processing: -

This is used to complete pattern discovery. This use is very crucial because there is only a bit of information is available like, user information, IP addresses, and site clicks.

2. Content Processing: -

This includes the change of Web data like pictures, content, scripts and others into helpful structures

3. Structure processing: -

Web Structure data - HTML or XML tags.

4. User Profile :-

Data that is user specific like registration and customer profile. In web usage mining for tracing the movement of the web pages, data mining techniques are applied. Usage mining extracts the navigation patterns as the browsing by the customer. It provides a cost effective way doing business.

Functions of web usage mining

i. The main function of Web Usage mining is used in business.

ii. WUM is also useful for E-commerce.

iii. Determining frequent access behavior for users

iv. Improve effectiveness of e-commerce (sales and advertising)

v. Personalization-tracking of previously archived pages. 




Figure 2. Process of Web usage Mining

\section{Recommendation System}

The Recommendation is a technique of web mining used to provide, the better or efficient result. There is the large amount of data on worldwide, Recommendation systems filter the data and provide efficient and user interested result. Proposal frameworks are universally used to prescribe items to the end clients that are generally proper. Recommender frameworks have been generally embraced by numerous online destinations/ administration as of late. These systems are an important mechanism for enabling users to contract with the massive information overload, since they provide suggestions of items/services, which are chosen in a way to match the user's preferences and interest.

Recommender systems (RS) play a significant role to enable us to make effective use of information.

For example, Amazon adopts a recommendation system for product recommendations, and Netflix uses recommendation system for movie recommendations. Existing RS methods can be generally ordered in three categories:

i. Content Based Filtering Method

ii. Collaborative Based Filtering Method

iii. Hybrid Method

In Content-based filtering technique [1] filtering is done based on customer's interested items. In content-based filtering technique, from the ancient database the web pages are recommended for a user very hastily. In that database different content of items are added that the user has used in the antique times and/or user's personal information and preferences. The client's information documents can be made by utilizing reactions to inquiries, thing appraisals, or the client's route data to surmise the client's inclinations and intrigues.Using this method, recommendation can be done mainly from the available database and past experience of the website visitor. The disadvantage of this method is, not all the time users give their ratings, proper for a website or web pages.

In collaborative filtering approach, [2] pages are prescribed to a specific client when other comparative sort of clients likewise lean towards those website pages For example, it may be defined as users having similar ratings of website pages or websites or users having related navigation behavior. Hybrid methods provide the functionality of both filtering content based and collaborative filtering method. 


\section{Literature Review}

Mohit Rajput et al.. proposed point by point examination of various methodologies in web personalization in view of various areas with their focal points and restriction. "Web personalization is the approach of customizing a site or substance of site to the prerequisites of each specific client or set of clients, taking profite of the data accomplished through the inspection of the client's navigational execution".[3] Some methods are based on the content of the website pages and users' interest \& some of the algorithm includes DM techniques and clustering.

Dipa dixit proposed two architectures for catch user instinct in the form of the recommendation list containing web pages which is visited by users and other user's those having similar usage profile. [4]

Ida Mele focused on two essential issues, first is to offer clients to discover recommending so as to intriguing web some assistance with paging blog entry and new articles, and second through static caching of search result in improving search engine performance. In this she proposed graph based approach for web pages recommendation and query covering approach static caching of search result. [5]

Neeraj Rahejaet al.. proposed an approach for WUM based upon web log partition. It takes less time and provides an appropriate result. In which web logs are used in cluster form. These records are designed according to user behavior records in web logs. Hence we do not need to search complete web logs, we search only from these clusters. [6].

Pooja Choudhary used search with synonyms as a challenging problem for Web search, as it could easily cause, intent drifting since synonym discovery is context sensitive. First is to offer clients to discover recommending so as to intriguing web some assistance with paging blog entry and new articles. On the off chance that we basically supplant them in hunt questions in all events, it is anything but difficult to trigger inquiry expectation floating. [7]

C. By Kun Xing et al.. "Behavior based User Interests Extraction Algorithm" . This paper proposed a method to extract user interest from user behavior history and document information. This paper proposed a technique to concentrate client enthusiasm for client conduct history and record data. In this paper, we will apply a three calculations Document data calculation is called conduct based client interest extraction calculation. How to get a user interest from the history of user behavior is an important issue. An experimental show BUIE algorithm could get better performance on user interest's extraction than those that don't consider user behavior.[8]

Marcelo G, et al.. proposed a thing suggestion calculation in light of idle component which utilizes certain input from clients to advance the positioning of thing as indicated by individual exhibitions. In this paper, we use two algorithms MABPR and MABPR SVD++.That algorithm improves the quality of the recommendation. Such decryption is an important source to construct a personalized set of items. The drawback is that these algorithm BPR and g SVD can not apply for explicit feedback, it only supports implicit feedback. So improve the quality of implicit feedback we can use the MABPR and $g$ SVD++ Extension. [9]

Yoon Ho Cho et al . has utilized choice tree affectation strategy, affiliation standard mining calculations and information warehousing innovations to take care of the issue of sparsity and versatility in collective sifting system. In view of that new half and half method has enhanced the proficiency of the cooperative using so as to separate approach web usage mining. Authors have used web logs as a database to find the frequent patterns using the Apriori algorithm. And to classify the customers author use Decision tree induction method [10]. 


\section{Comparison Between Various Work}

\begin{tabular}{|c|c|c|c|}
\hline Related Work & Authors & Merits & Den \\
\hline $\begin{array}{l}\text { Automatic } \\
\text { Recommendation } \\
\text { for Online Users } \\
\text { Using Web Usage } \\
\text { Mining }\end{array}$ & $\begin{array}{l}\text { Ms.Dipa } \\
\text { Dixit, } \quad M r \\
\text { Jayant } \\
\text { Gadge }\end{array}$ & $\begin{array}{l}\text { 1. The preference is given } \\
\text { to the user. } \\
\text { 2. Accuracy of the } \\
\text { recommender system is } \\
\text { high. } \\
\text { 3. Two tier architecture is } \\
\text { given which gives a better } \\
\text { view. }\end{array}$ & $\begin{array}{l}\text { 1. Complexity of the } \\
\text { system } \\
\text { recommended is } \\
\text { high. } \\
\text { 2. The system works } \\
\text { on the user's } \\
\text { institution, which is } \\
\text { not always correct. }\end{array}$ \\
\hline $\begin{array}{lr}\text { Behavior based } \\
\text { User Interests } \\
\text { Extraction } \\
\text { Algorithm }\end{array}$ & $\begin{array}{l}\text { Kun Xing, } \\
\text { Bofeng } \\
\text { Zhang, Bo } \\
\text { Zhou, } \\
\text { Yucong Liu }\end{array}$ & $\begin{array}{l}\text { 1. A user model which } \\
\text { satisfies maximum user } \\
\text { needs is built on this } \\
\text { research. } \\
\text { 2. The user interest is } \\
\text { consistent. }\end{array}$ & $\begin{array}{l}\text { 1.A slow technique } \\
\text { to be implemented. } \\
\text { 2. It scans whole } \\
\text { history to find user } \\
\text { behavior which } \\
\text { consumes time. }\end{array}$ \\
\hline $\begin{array}{l}\text { Optimizing } \\
\text { Personalized } \\
\text { Ranking in } \\
\text { Recommender } \\
\text { Systems with } \\
\text { Metadata } \\
\text { Awareness }\end{array}$ & $\begin{array}{l}\text { Marcelo G. } \\
\text { Manzato, } \\
\text { Marcos A. } \\
\text { Domingues, } \\
\text { Solange O. } \\
\text { Rezende }\end{array}$ & $\begin{array}{l}\text { 1. This system takes user } \\
\text { feedback which somehow } \\
\text { gives the actual rating and } \\
\text { a real world view to the } \\
\text { algorithm. } \\
\text { 2. A personalized set of } \\
\text { items is formed which } \\
\text { relates to user's interests. }\end{array}$ & $\begin{array}{l}\text { 1. The rating given } \\
\text { by the user's lead to } \\
\text { one-sided results as } \\
\text { the opinion of a } \\
\text { limited group is } \\
\text { taken rather than } \\
\text { more people. }\end{array}$ \\
\hline $\begin{array}{l}\text { An Improved Page } \\
\text { Rank Algorithm } \\
\text { based on } \\
\text { Optimized } \\
\text { Normalization } \\
\text { Technique }\end{array}$ & $\begin{array}{l}\text { Hema } \\
\text { Dubey, } \\
\text { N. Roy }\end{array}$ & $\begin{array}{l}\text { 1. HITS concept is used } \\
\text { which works for page } \\
\text { ranking. } \\
\text { 2. This normalization } \\
\text { technique works on mean } \\
\text { values of page ranks. } \\
\text { 3. Time complexity is } \\
\text { reduced. }\end{array}$ & $\begin{array}{l}\text { 1. Multiple links may } \\
\text { lead to a network } \\
\text { which may consume } \\
\text { time. } \\
\text { 2. Link structure of } \\
\text { the pages is } \\
\text { mandatory. }\end{array}$ \\
\hline
\end{tabular}

\section{Proposed Work}

The proposed work consists of the idea of creating a new RS based on page ranking that can be implemented in WM. The page rank of the websites can be reduced or increased with the help of data mining and how data mining can help in optimization of the ranking of the websites. The new proposed is based on this idea that it can implement DM in creating a web log and finding the websites based on the search of the keywords that are connected to the database in a way.

The entire procedure of the proposed calculation can be clarified in the accompanying way:-

1. First creating a database which contains the page rank, website name and keywords that relate to the website in a way. The second database is created which contains the synonyms of each keyword.

The ranking in the database is inserted on the basis of the algorithm designed by "google.com". The algorithm of page ranking on the Google is a standard one and is used as the base for giving the page rank. 
2. Applying an improved algorithm for finding the page rank. The sites are obtained that match the keywords and the synonyms from both the databases. The data is filtered based on the keywords and synonyms.

3. Arranging all the urls and the website names preferred.

4. Ranking is given by the user.

5. Update the ranking of the website. Then it shows the message. The ranking of the website is updated in the database.

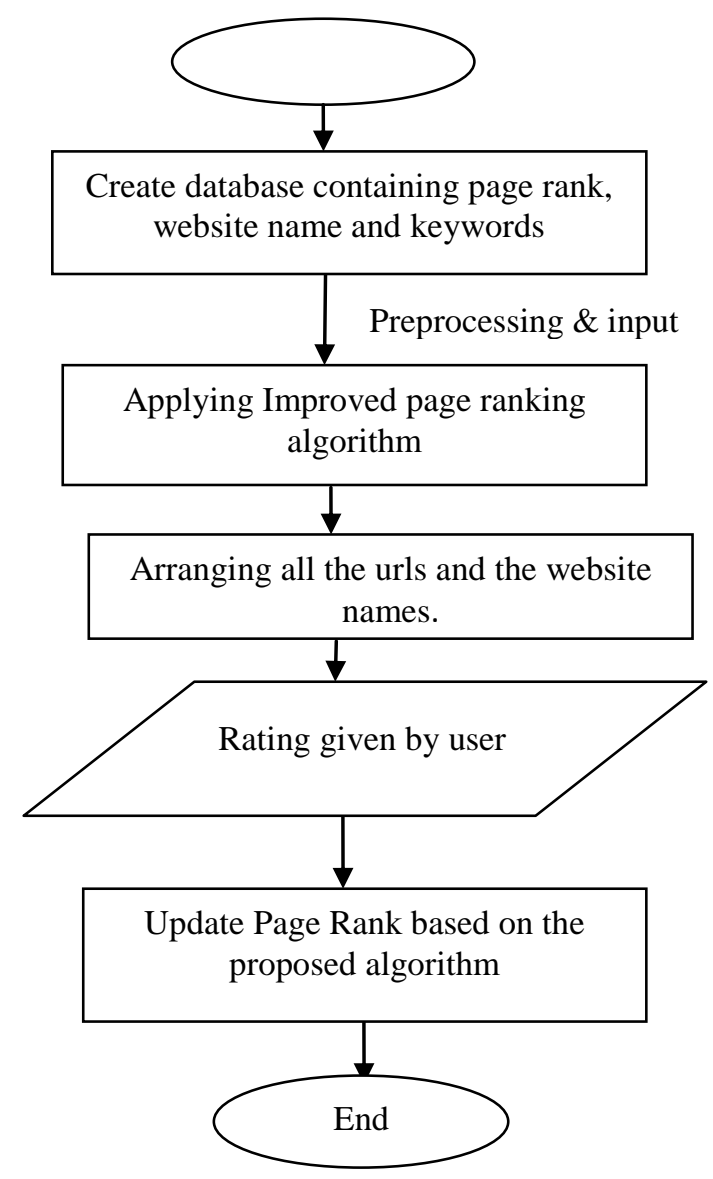

Figure 3. Proposed Flowchart

\section{Conclusion}

The Internet is the hottest region in Computer field. Billions of individuals every day getting to the web for looking a few sets of data. WM perform a significant role in discovering frequent pattern from WWW which is contain huge amount of data, but each time it does not provide authentic data, so we use web mining that rarefy data. WM is to use the DM approach to mining and uncover data from the web documents services, i.e. web data. Online shopping systems use the web mining to dig up the information of a product and its constraint during web mining. Web search makes the user to search over 2 billion data. It maintains the ranks among the pages and commercial ordering and circulate based on the user query. Along these lines, web server contains the immense measure of information consistently. Web Usage Mining encourages to investigate the Web Log Files. Some research is still needed to improve the efficiency of the algorithms to facilitate the website visitors, website analyst and website personalization. The proposed work will concentrate on the above said measures in a better manner. 


\section{References}

[1] N. Paritosh and A. Thomas, "Survey on recommendation system methods", 2nd International Conference In Electronics and Communication Systems (ICECS), IEEE, (2015), pp. 1496-1501.

[2] R. Doug, "Personalized views of personalization", Communications of the ACM 43, no. 8, (2000), pp. 27-28.

[3] R. Mohit, R. Agrawal and D. K. Sharma, "A study and comparative analysis of web personalization techniques", IEEE Conference on Information \& Communication Technologies (ICT), (2013), pp. 12931298.

[4] D. Dixit and Gadge, "Automatic Recommendation for Online Users Using Web Usage Mining”, arXiv preprint arXiv:1009.0433, (2010).

[5] M. Ida, "Web usage mining for enhancing search-result delivery and helping users to find interesting web content", In Proceedings of the sixth ACM international conference on Web search and data mining, (2013), pp. 765-770.

[6] R. Neeraj and V. K. Katiyar, "Efficient Web Data Extraction Using Clustering Approach In Web Usage Mining”, IJCSI International Journal of Computer Science Issues, vol. 11, no. 2, (2014), pp. 216225.

[7] C. Pooja, "A Comparative Analysis of Various Web Search Engines", International Journal of Computing and Business Research (IJCBR), vol. 3, no. 2, (2012).

[8] X. Kun, B. Zhang, B. Zhou and Y. Liu, "Behavior based user interests extraction algorithm", 4th International Conference on Cyber, Physical and Social Computing, In Internet of Things (iThings/CPSCom) IEEE, (2011), pp. 448-452.

[9] G. M. Marcelo, M. A. Domingues and S. O. Rezende, "Optimizing personalized ranking in recommender systems with metadata awareness", In Proceedings of the IEEE/WIC/ACM International Joint Conferences on Web Intelligence (WI) and Intelligent Agent Technologies (IAT), vol. 1, (2010), pp. 191-197.

[10] C. Y. Ho, J. K. Kim and S. H. Kim, “A personalized recommender system based on web usage mining and decision tree induction", Expert systems with Applications Elsevier Science, vol. 23, no. 32, (2002), pp. 329-342. 